\title{
In vitro synergy of sertraline and tetracycline cannot be reproduced in pigs orally challenged with a tetracycline resistant Escherichia coli
}

Sofie Kromann, Anna Hvidtfeldt, Mette Boye, Dorte Bratbo Sørensen, Steffen Jørgensen, Jens Peter Nielsen and Rikke Heidemann Olsen ${ }^{*}$ (1)

\begin{abstract}
Background: Antimicrobial helper-compounds may reverse antimicrobial resistance. Sertraline, a antidepressant drug, has been suggested as a tetracycline helper-compound. Tetracycline is the preferred antimicrobial for treatment of enteric diseases in pigs. This study is the first to evaluate the potency of sertraline as a tetracycline adjuvant in pigs.

Methods: Forty-eight nursery pigs were divided into four treatment groups: Tetracycline, sertraline, tetracycline/sertraline or un-medicated control. Fecal and ileal samples were obtained before treatment, $48 \mathrm{~h}$ and nine days after five days of treatment, respectively. Colony forming units (CFU) of tetracycline resistant coliforms in each sample (ileal or fecal) and CFU of an orally inoculated tetracycline-resistant strain of Escherichia coli were determined at each sampling point. The microbiome of fecal and ileal and samples was analyzed by sequencing of the 16S V3-V4 region.

Results: The results did not provide evidence that sertraline in combination with tetracycline has any impact on tetracycline resistant bacteria in either fecal or ileum samples, while in the tetracycline treated group of pigs, an increase in the prevalence of a tetracycline resistant indicator strain of Escherichia coli shortly after ended five-day treatment was observed. The ileal samples obtained shortly after ended treatment showed treatment-associated changes in the composition of the microbiota in the groups of pigs treated with tetracycline (+/-) sertraline. While tetracycline treatment increased the abundance in the reads of $E$. coli, sertraline/tetracycline treatment led to increased abundances of Streptococcus spp. and decreased abundances of Lactobacillus spp. However, all observed differences (on CFU counts and microbiota composition) between groups shortly after treatment had diminished in less than two weeks after last treatment day.

Conclusions: Sertraline (+/-) tetracycline treatment did not reduce the long-term level of tetracycline-resistant bacteria in the feces or small intestine contents of piglets compared to the un-medicated control group of pigs. The result of this study reflects the importance of in vivo studies for confirmation of the antimicrobial helper-compound potential of an in vitro active compound.
\end{abstract}

Keywords: Antimicrobial resistance, Tetracycline, Sertraline, Synergy, Pigs, E. coli

\footnotetext{
* Correspondence: cava@sund.ku.dk

Department of Veterinary and Animal Sciences, Faculty of Health and

Medical Sciences, University of Copenhagen, Frederiksberg, Denmark
}

(c) The Author(s). 2019 Open Access This article is distributed under the terms of the Creative Commons Attribution 4.0 International License (http://creativecommons.org/licenses/by/4.0/), which permits unrestricted use, distribution, and reproduction in any medium, provided you give appropriate credit to the original author(s) and the source, provide a link to the Creative Commons license, and indicate if changes were made. The Creative Commons Public Domain Dedication waiver (http://creativecommons.org/publicdomain/zero/1.0/) applies to the data made available in this article, unless otherwise stated. 


\section{Background}

For many years tetracyclines have been the most commonly used antimicrobials to treat pigs with enteric disease [1]. The widespread uses of tetracyclines have been suggested as the major cause of the increase in tetracycline resistant bacteria, in particular in pigs [2]. Tetracyclines have been favored in agriculture because of its broad-spectrum activity, low toxicity, many formulations for oral use and the relative low price $[3,4]$, but the need to bring down the amount of antimicrobials used in the livestock industry is inquisitional to inhibit further emergence of antimicrobial resistance $[5,6]$. In addition, antimicrobial helper-compounds have received increased focus. Helper-compounds may be described as drugs that enhance the activity of antibiotics [7]. Some helper-compounds possess antimicrobial activities themselves [7], while others interfere with the resistance mechanism of the bacterial resistance, e.g. by blocking antimicrobial efflux pumps [8]. Sertraline, a selective serotonin reuptake inhibitor (SSRI), is a medical compound normally prescribed for human mental disorders, such as depression and anxiety, and it has been suggested as tetracycline helper-compound by several research groups [9-13]. Although sertraline itself do possess antimicrobial activity against a number of Gram-negative bacterial species, the main reason of interest for sertraline as a helper-compound is due to the interference with the tetracycline resistant efflux pump, TetA [10, 13]. While several in vitro results have been published on the antimicrobial helper-drug activities of SSRIs, including sertraline, only very limited literature on the clinical in vivo effect of sertraline's helper-compound activity exist.

The aim of the present study was to evaluate the influence of sertraline on the CFU of intestinal commensal tetracycline coliforms in concurrent tetracycline treated piglets compared to the level in non-medicated, sertraline - or only tetracycline treated groups of pigs. For the same groups, the impact of treatment on the ileal microbiota composition was assessed.

\section{Results \\ Level of tetracycline resistant bacteria in feces before inoculation}

Two days before treatment start and just prior to inoculation of all pigs in all four groups (Time point $\mathrm{T}[-2]$ the level of fecal tetracycline resistant coliforms in the 48 individual fecal samples ranged from $3.3-7.7 \log _{10}$ CFU per gram of feces (Fig. 1). None of the fecal samples contained bacteria which could be cultured on the indicator agar plate (MacConkey agar supplemented with tetracycline, ampicillin and rifampicin).

Level of tetracycline resistant bacteria in feces and ileum immediately before treatment start

Two days after inoculation with the indicator bacteria (Time point $(\mathrm{T}[0])$, fecal samples were obtained from all
48 pigs, while ileal samples were obtained from two pigs per treatment group. At $\mathrm{T}[0]$ the indicator bacteria could be re-isolated (confirmed by PCR) in all fecal samples, while for one out of eight pigs the indicator bacteria could not be detected in the ileal samples at this time point. The level of the indicator E. coli at T[0] in fecal and ileum samples varied between 4.3-6.3 $\log _{10}$ to 0-4.1 $\log _{10} \mathrm{CFU} / \mathrm{g}$, respectively, (Fig. 2). There was no significant difference between the groups in the level of the indicator E. coli at T[0] (Fig. 2,). Overall, in paired fecal and ileum samples from the same pig there were lower CFU counts observed in the ileum samples.

\section{Level of tetracycline resistant bacteria in fecal/ileal samples after treatment}

Forty-eight hours after the last of five treatment days, fecal samples were obtained from all pigs in each group (Time point T[7]). Hereafter, five pigs from each group were randomly chosen for euthanization and from these pigs ileum samples were obtained as well. Nine days later, fecal and ileum samples were obtained from the remaining pigs in each group (Time point $\mathrm{T}[15]$ ).

At T[7] there was significantly higher count of tetracycline resistant coliform CFU per gram in fecal samples obtained from tetracycline, sertraline or sertraline/tetracycline treated pigs compared to the CFU per gram fecal samples obtained from un-mediated control pigs (Fig. 1). Similarly, ileum samples from un-mediated control pigs contained significantly lower tetracycline resistant coliform CFU per gram compared to the tetracycline or sertraline treated group at T[7] (Fig. 1).Ileum samples from the tetracycline treated group (Group 2) had a significant higher level of the indicator $E$. coli compared to the level in any of the other three groups (Fig. 3), while there were no difference between the groups in the level of the indicator bacteria in fecal samples (Fig. 2).

At $\mathrm{T}[15]$ there was no statistical significant difference in any CFU counts between the four groups, whereas there were several difference in CFU counts within each of the groups (Fig. 1). There was a significant decrease in total fecal tetracycline resistant coliform CFU per gram at time $\mathrm{T}[15]$ compared with $\mathrm{T}[7]$ for the tetracycline treated group (Group 2), the sertraline treated group (Group3) and sertraline/tetracycline treated group (Group 4), respectively (Fig. 1), while the were no difference in the level of fecal tetracycline resistant coliform CFU in the control group. The level of the indicator E. coli in feces also decreased significantly from time $\mathrm{T}[0]$ to time $\mathrm{T}[15]$ in the tetracycline and sertraline/tetracycline treated groups (Fig. 2).

\section{Microbiota sequencing results}

Sample preparation and sequencing were successful for 67 out of 68 samples (quality of sample preparation of DNA from a fecal sample from a pig in the tetracycline-treated 


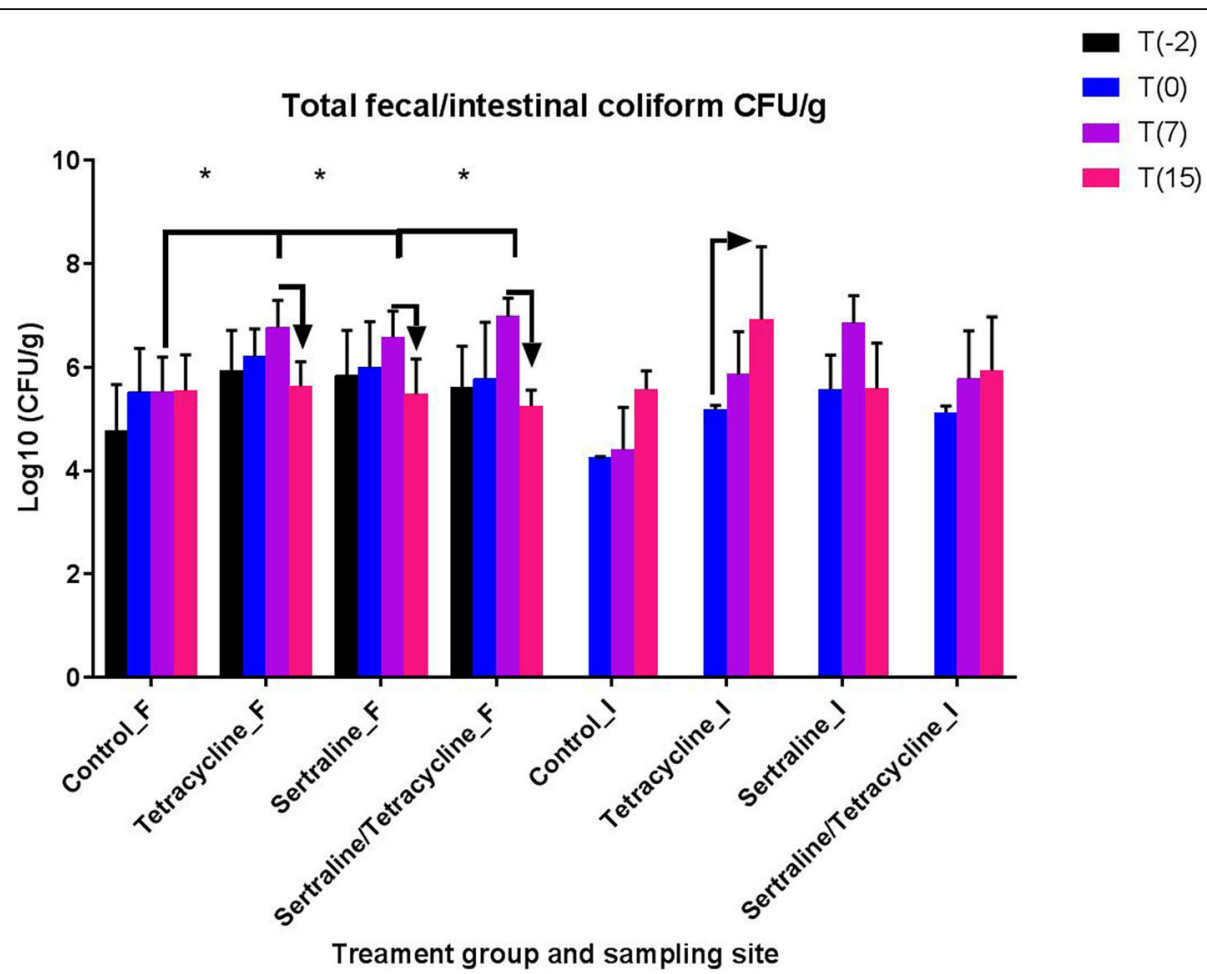

Fig. 1 Total fecal (_F) and intestinal (_I) tetracycline resistant CFU Numbers (in $\log _{10}$ CFU per gram feces (_F) or ileum (_I)) of total tetracycline resistant CFU at different time points in four different treatment groups. Vertical bars indicate standard deviations. Timepoint $T(-2)$ refers to two days before treatment start, and just prior to oral inoculation of all pigs with a tetracycline/rifampicin resistant Escherichia coli strain. At T(- 2) 12 individual fecal samples were obtained from each group. No pigs were euthanized at this point, therefore no ileum samples were obtained at $\mathrm{T}(-2)$. Time point $\mathrm{T}(0)$ refers to the first treatment day. Samples were obtained prior to the first treatment at the same day. From each group 12 individual fecal samples were obtained. Hereafter, two pigs were euthanized at ileum samples were obtained from 2X4 pigs. Time T(7) refers to $48 \mathrm{~h}$ after the last of five days of treatment. From each group 10 individual fecal samples were obtained. Hereafter, five pigs were euthanized and ileum samples were obtained from 5X4 pigs. Time T(9) refers to nine days after the last of five days of treatment. From each group five individual fecal samples were obtained. Hereafter, the five pigs in each group were euthanized and ileum samples were obtained from $5 \times 4$ pigs. For visual purposes all CFU counts at the different time points has been included in the same figure, statistical analysis were, however, done for each time point, each treatment group at different sampling time and sampling site (fecal or ileum) individually. Asterisks over straight lines indicate a statistical difference at the same time point between treatment groups. Arrows indicate statistical difference at the different time points within each treatment groups. Hash tag indicates that group is statistically different from the other groups at the same sampling time. Statistical significant differences between fecal and ileum samples are not shown on the figure

group obtained nine days post ended treatment did not pass quality control). The number of reads, OTU and Shannon index per sample is stated in Additional file 1: Table S1. The average Shannon index was significantly lower in ileum samples (average 1.78 for all ileum samples combined) then the fecal samples (4.62). There were no significant differences in the Shannon indexes between ileal samples from different treatment groups or different sampling times.

The impact of treatment and time post treatment on the ileum microbiota composition

Ileum samples were obtained from two, five and five pigs at time points $\mathrm{T}[0], \mathrm{T}[7]$ and $\mathrm{T}[15]$, respectively. Before treatment start, the samples had a large diversity in microbiota composition (Fig. 3). At time T[7] a treatment-associated clustering of ileum microbiota samples from tetracycline- and sertraline/tetracycline treated groups was observed, while there were no distinct clustering of ileum samples from pigs in either control or sertraline treated groups (Fig. 3). For samples obtained at time T[7] the abundance of the Lactobacillaceae had the most distinct differences between groups. The level of the Lactobacillaceae had a significantly lower abundance in the tetracycline and sertraline/tetracycline treated groups compared to the level in the samples from the un-medicated control pigs. Furthermore, the abundance of the Lactobacillaceae was significantly lower in the sertraline/tetracycline treated group compared to samples from the tetracycline- or sertraline-treated groups. In contrast, the abundance of the Streptococcaceae in the control group was significantly higher than the abundance of Streptococcaceae in the tetracycline and sertraline/tetracycline treated groups. Furthermore, the abundances of Streptococcaceae were significantly lower in the sertraline/tetracycline compared to 


\section{Indicator Esherichia coli CFU/g}

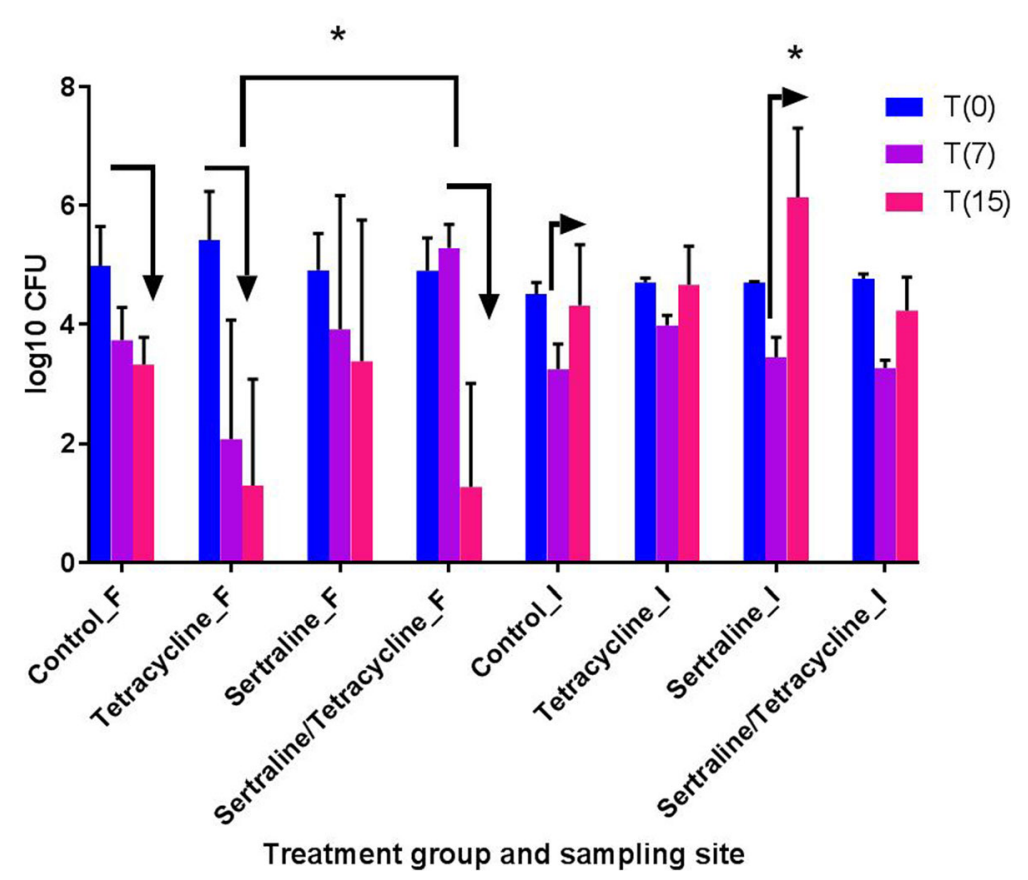

Fig. 2 Indicator E. coli CFU Numbers (in $\log _{10}$ numbers of CFU per gram feces (_F) or ileum (_I)) of an indicator E. coli strain at three different time points in four different treatment groups. Vertical bars indicate standard deviations. Before the pigs were inoculated with the indicator strain, no pigs carried a detectable level of the indicator strain in feces (data not shown on the figure). Time point $\mathrm{T}(0)$ refers to the first treatment day. Fecal and ileum samples were obtained prior to the first treatment at the same day. From each group 12 individual fecal samples were obtained. Hereafter, two pigs were euthanized and ileum samples were obtained from $2 X 4$ pigs. Time T(7) refers to $48 \mathrm{~h}$ after the last of five days of treatment. From each group 10 individual fecal samples were obtained. Hereafter, five pigs were euthanized at ileum samples were obtained from 5X4 pigs. Time T(15) refers to nine days after the last of five days of treatment. From each group five individual fecal samples were obtained. Hereafter, the five pigs in each group were euthanized and ileum samples were obtained from 5X4 pigs. For visual purposes all CFU count at the different time points has been included in the same figure, statistical analysis were, however, done for each time point and sampling site individually. The asterisk indicate that the ileum samples from the tetracycline treated group had a significant higher level than ileum samples from the three other groups at time point $\mathrm{T}(7)$

samples from the tetracycline and sertraline treated groups (Fig. 4, Additional file 2: Figure S2). The average abundance of Enterobacteriaceae in ileal samples from the tetracycline-treated group had a significantly higher average abundance of Enterobacteriaceae compared to any of three other groups (Fig. 4). For the remaining seven out of the ten overall most abundant families in the ileum samples (Fig. 4), there were no significant differences in the abundances between groups. At time point $T[15]$, there were no longer any significant differences in the abundances of the 10 most abundant families across all ileal samples between the different treatment groups (Fig. 4), and no treatment-associated clustering of the ileum derived microbiota-samples (Additional file 3: Figure S1).

\section{Average daily weight gain between groups}

The average daily weight gain per pig per group varied between $0.4-0.8 \mathrm{~g} /$ day. There was no statistical difference between the groups in average daily weight gain per group.

\section{Discussion}

The main purpose of the study was to investigate if sertraline would re-sensitize an intestinal tetracycline-resistant population of bacteria, in particularly coliforms, to tetracycline in piglets. Secondly, the question as to how oral treatment with sertraline with or without tetracycline may impact the gut microbiota composition in relation to treatment and nine days after withdraw of medication, was studied.

Initially, there are some methodic issues of the study to address; First, as regulation of the European Union does not allow tetracyclines, or any other antibiotics, as in-feed growth promoters [14], the use of tetracycline in agriculture within EU is always related to a treatment-related aspect. In pig production tetracycline is the most commonly used antibiotic for treatment of enteric diseases [1]. Therefore, it could be argued if an experimental diarrhea model in pigs would have been more appropriate to address the question of helper-drug activity of sertraline. Such model is, however, fairly difficult to establish 


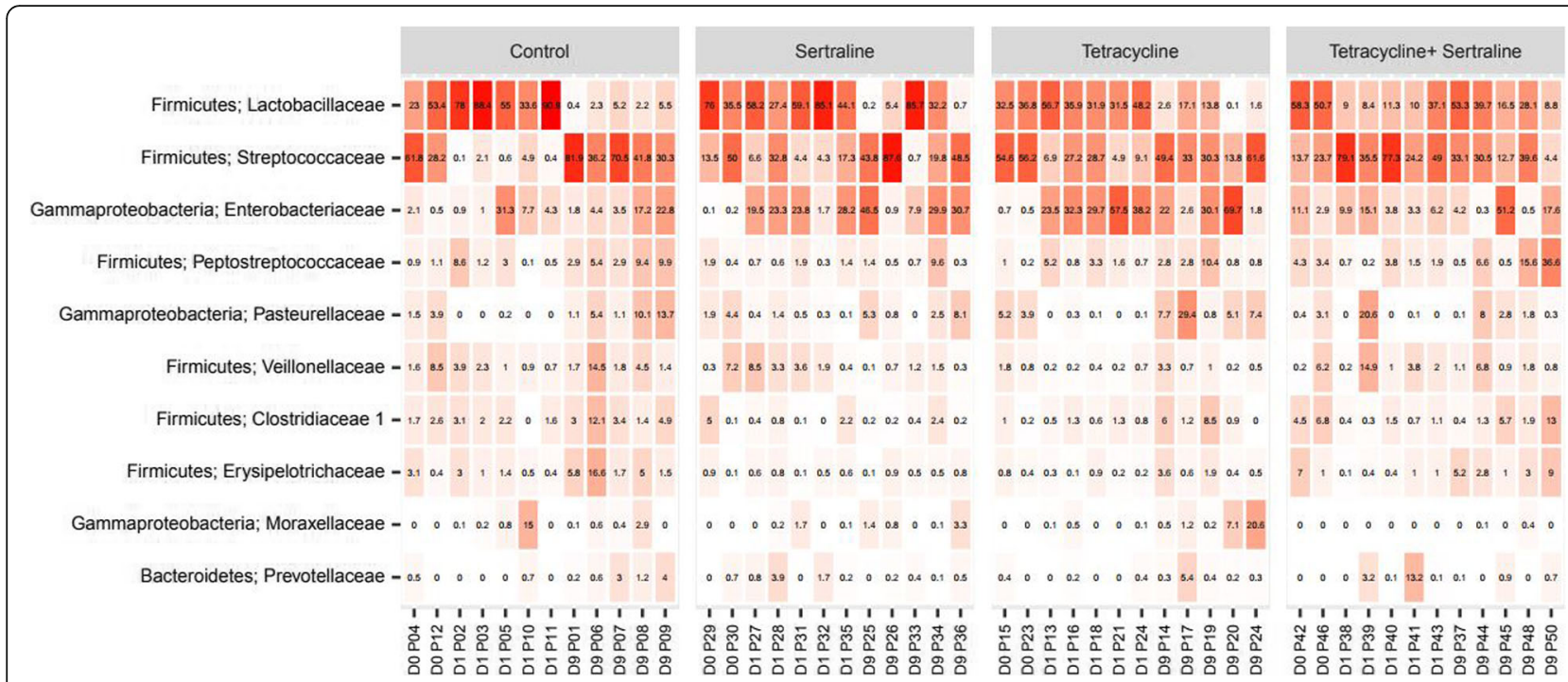

Fig. 3 Principle component analysis (PCA) of ileum microbiota composition at time T[0] and T[7] Identification of samples with similar microbiota composition using multivariate statistics (PCA). Each red or blue point represent the microbial community in a specific sample obtained just prior to treatment start $(\mathrm{D} 0 / \mathrm{T}[0])$ or $48 \mathrm{~h}$ after last day of treatment $(\mathrm{D} 1 / \mathrm{T}[7])$, respectively

experimentally with success [15]. Often, only a limited amount of the pigs would develop diarrhea, likely because porcine enteritis is a complex disease with several factors contributing to diarrhea development [16]. Secondly, since all pigs were initially colonized with tetracycline resistant bacteria before inoculation with the indicator E. coli bacteria, this step could perhaps have been omitted. However, to ensure that each pig would have an equal challenge of a marked tetracycline-resistant bacteria, in which sertraline and tetracycline synergy had been confirmed in vitro [10], the inoculation step remained in the experimental design. Thirdly, the concentration of sertraline used to obtain in vitro synergy with tetracycline, is considerable higher than the maximal (human) plasma concentrations of sertraline $(0.25 \mathrm{mg} / \mathrm{L})$ [17], and therefore negative in vivo results may have been expected. However, to the authors' knowledge there is no literature available on the concentration of sertraline in intestine of either animals or humans after oral medication. In the present study, we used daily doses of sertraline that would correspond to the maximum dose given to a person with severe mental disorders e.g. severe anxiety. Higher doses of sertraline may cause a general immune suppression, which increase the risk of infections [18]. Finally, even though tetracycline are used less frequently in human medicine than in agriculture, the high carriage of commensal tetracycline resistant bacteria in food-producing animals do possess a public risk, in particular because tetracycline resistance is likely to co-select for other, more human relevant antibiotics [19], therefore we find it relevant to investigate synergy of sertraline/ tetracycline on the tetracycline-resistant commensal microbiota in pigs. Based on these considerations, we find the experimental design and treatment doses used appropriate to address the research questions raised above.

The heavily fecal shedding of tetracycline resistant bacteria in all pigs before the experimental inoculation (Fig. 1) is most likely caused by the intensive use of antibiotics in the pig industry in the last decades, as at a population level there is a strong association between the use of antibiotics and the proportion of bacteria resistant to antibiotics $[5,20,21]$.

For all treatment groups, the treatments were tolerated well by the pigs with no clinical signs despite that a high-dose sertraline were administered to two of the treatment groups (Group 2 and 4). Toleration of the clinical relevant concentrations of the helper-drugs by the animals (or humans) is probably underrated [13] e.g. in another recent published study evaluating in vivo helper-compound activity of thioridazine, also a neurotrophic active medical compound, the authors report that their study had to pre-terminate due to severe side-effects of thioridazine in pigs with the concentrations used [22].

As expected, the pigs did not show clinical affection of the high-dose inoculum indictor bacteria. Fecal shedding of the indicator bacteria was very high two days after inoculation (Fig. 2), and a part of the inoculum there has probably mainly transited the intestinal tract with a only minor degree of colonization in ileum at $\mathrm{T}[0]$ (Fig. 2). For the tetracycline and sertraline/treated groups the fecal shedding of the indicator bacteria decreased significantly from time $T[7]$ to $T[15]$, while level in ileum remained at the same level as the level at $T[0]$ for all 


\section{Ileum samples 1 day after ended treatment}

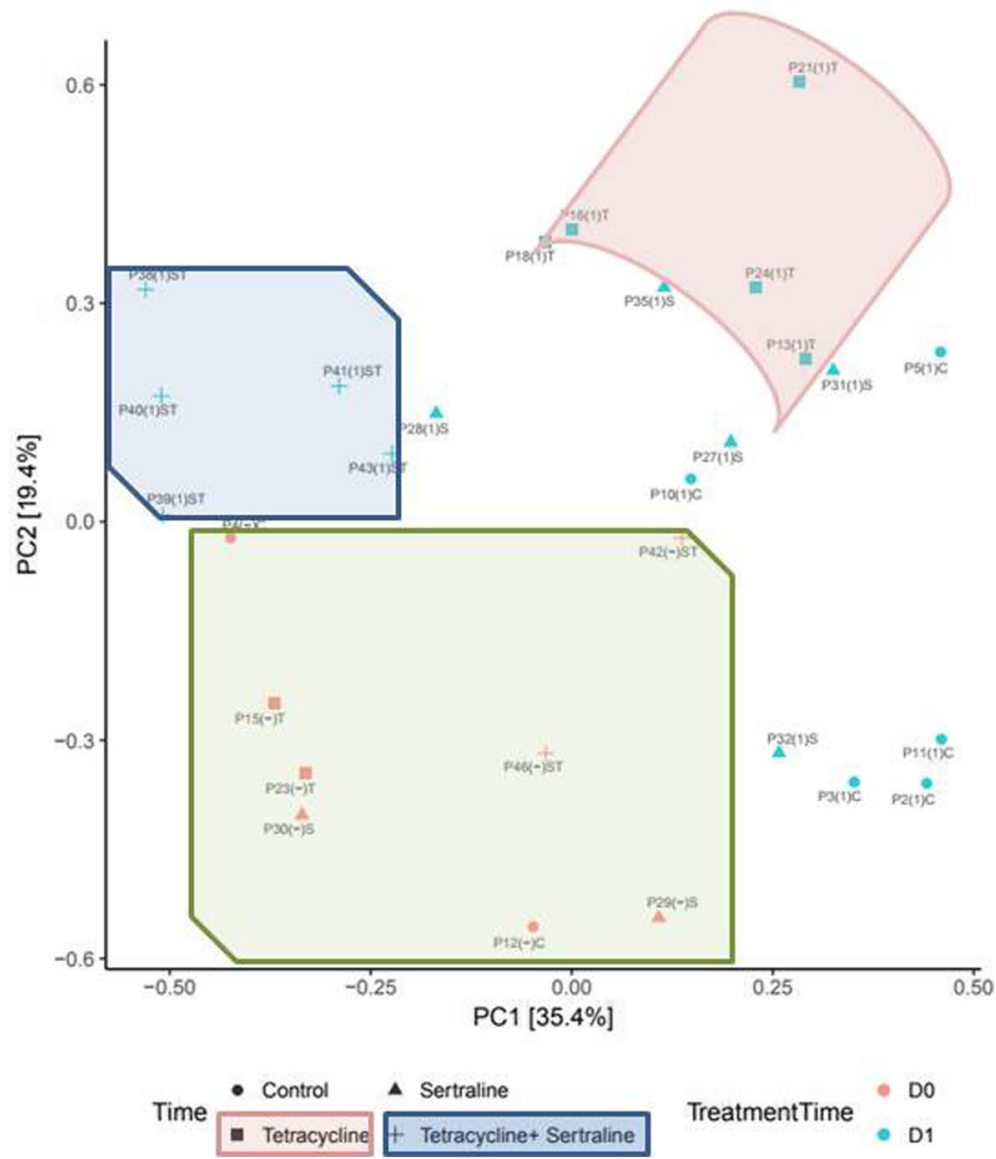

Fig. 4 The 10 most abundant families in ileum samples obtained at time T[0], T[7] and T[15] The 10 most abundant genera in percent. Each has both a broad name (Phylum) and a specific name (Family). Samples were obtained from each treatment just prior to treatment start (D0/T[0]), 48 $\mathrm{h}$ after last day of treatment (D1/T[7]) and nine days after last day of treatment (D9/T[15])

groups (Fig. 2) indicating that some degree of intestinal colonization had occurred. For the tetracycline treated group, but not the sertraline/tetracycline, there was a significant increase in the intestinal level of the tetracycline resistant indicator $E$. coli strain shortly after withdrawal of tetracycline treatment (Fig. 2). This could indicate that sertraline may counteract the selective pressure of tetracycline, however, the level of the indicator $E$. coli remained as high as the average level of the indicator bacteria in ileal samples from the un-medicated control (3.8 $\log _{10}$ CFU) (Fig. 2). Furthermore, within the group of sertraline/tetracycline treated pigs, there was a rise in the number of total tetracycline resistant coliform bacteria at $\mathrm{T}$ [7] compared to control group (Fig. 1), hence, the suggested counteracting of a selective pressure, indicated by the lower level of resistant bacteria in the sertraline/tetracycline compared to tetracycline group, is not likely to have clinical impact. The difference between the impact of sertraline/tetracycline on the level of the indicator $E$. coli bacteria and the lack of impact on total tetracycline resistant coliforms could possibly be explained by the various types of tetracycline resistance [23], of which tetracycline and sertraline synergy has only been documented for the efflux-mediated type of tetracycline resistance, a mode of action present in the indicator E. coli strain [10].

The results on the selective pressure on the indicator bacteria is in contrast with the observations on the total tetracycline resistant coliforms at T[7], in the fecal samples. The fecal samples from tetracycline and sertraline/ tetracycline treated groups had similar levels of tetracycline resistant coliforms, and the level tetracycline resistant coliforms of these two groups was significantly higher than the control group level at T[7] (Fig. 1). Similarily, theof intestinal tetracycline resistant coliforms was also significantly higher in the tetracycline treated group compared to the control group (Fig. 1). The finding of tetracycline treatment may select for a higher level of resistant bacteria followed by a return to the level before 
medication in a time span (in our study, nine days) from last day of short-time antibiotic treatment (Fig. 1)) is in accordance with previous observations [24]. However, nine days after ended treatment both the tetracycline and sertraline/tetracycline treated groups had levels of fecal tetracycline resistant coliform comparable to the un-medicated control group, implying that sertraline do not potentiate the effect of tetracycline in a population with a high proportion of commensal, tetracycline resistant bacteria. For the intestinal samples, however, the tetracycline treated group was the only group in which the level of tetracycline resistant coliforms remained significantly higher nine days after treatment compare to the level before treatment for the same group (Fig. 1). The level of tetracycline resistant coliforms were, nevertheless, not higher in the tetracycline group at nine days after ended treatment compared to the other treatment/ control groups. Taken together, these results of the present study confirm a recent study by Graesboll et al. [24] reporting that five days treatment with tetracycline may transiently result in increased total count and proportion of tetracycline resistant coliform (Fig. 1).

In agreement with the significantly increased level of tetracycline resistant bacteria right after ended treatment for tetracycline (+/- sertraline) treated groups, treatment-associated grouping of ileal microbiota samples of pigs from Group 2 (tetracycline treated) and Group 4 (sertraline/tetracycline treated) were found (Fig. 3). Samples from Group 2 and 4 assembled individually, while samples from un-mediated control pigs and sertraline treated pigs did not demonstrate treatment-associated microbiota uniformities. The different compositions of microbiotas indicate that sertraline combined with tetracycline has an interactive effect that is different from the impact of the sum of changes caused by individual treatment with either sertraline or tetracycline, proposing that there might be some synergy or at least interaction between tetracycline and sertraline on the microbiota composition in vivo.

Nine days after ended treatment there were no indication of treatment-associated clustering of ileum-derived microbiotas (Additional file 3: Figure S1), corresponding to the observations done on comparisons of CFU counts from the different treatment groups.

The amount on literature concerning the microbiota of animals as humans has increased almost exponentially the last decade. Yet, the optimal intestinal microbiota composition is yet to be described. Nevertheless, a recent publication combining metagenomics and microbial function genetics and the impact of in-feed antibiotics has enlighten the understanding on how a shift in bacterial population may also shift energy production and conversion and, hence, growth of the pigs. In the above mentioned study, feeding pigs with a commercial available (in the US) performance-enhancing mix of antibiotics lead to a bacterial shift, dominated by an increase in phylum Proteobacteria, in particular E. coli, in the medicated pig compared to the un-medicated control [25]. These observations are in agreement with the present study, in which a significant increase in the abundance of $E$. coli in ileal samples from the tetracycline-treated pigs compared with ileum samples from the un-treated control pig (Additional file :3 Figure S1). The unique increase in the abundance of E. coli in the tetracycline treated group at time point $\mathrm{T}[7]$ is in agreement with the unique increase of the CFU per gram of indicator $E$. coli at the same time point. The same increases in either abundance or CFU count of $E$. coli were, however, not evident in the ileum samples from the pigs treated with tetracycline in combination with sertraline (Fig. 1 Fig. 4). Rather, for the latter mentioned group the level of the family Streptococcaece was significantly increased, while the abundances of the family of Lactobacillaceae was dramatically decreased at time point T[7]. Lactobacillus spp. are probably the most treasured bacteria in term of general health-promoting probiotic effects [26], and hence a low level of lactobacillus would be considered strongly un-favorable. It can be concluded that sertraline and tetracycline have an interaction that create a measurable response on CFU per gram and microbiota composition right after ended treatment, although the effect may neither be major (in terms of decreasing the level of tetracycline resistant coliforms) nor beneficial (in terms of an optimal microbiota composition). However, in accordance with the observations done on the cultivable bacteria, the microbiota compositions nine days after withdrawal of treatment did no longer show any association with any previous treatments (Fig. 4, Additional file 2: Figure S1), underling that the five days of treatment with tetracycline in therapeutic concentrations (+/- sertraline) did not have long-lasting effects on the intestinal bacterial population, including the tetracycline resistant population.

\section{Conclusion}

The high carriage of antimicrobial resistance bacteria in the porcine autochthonous microbiota calls for new treatment strategies to ensure continuous treatment success and human food safety. In this study, the impact of sertraline as a tetracycline helper-compound had only very limited effect and is not likely to have a clinical importance. Furthermore, the increased level of tetracycline-resistant coliforms in the tetracycline-medicated group (without sertraline added) shortly after ended treatment returned to a level comparable to the un-medicated control group in less than two weeks after ended treatment. Similarly, the composition of ileal microbiota from tetracycline and tetracycline/sertraline medicated groups, clustered according to treatment only immediately after treatment. Nine days after ended treatment no treatment-associated effect could be detected. 
In conclusion under the conditions tested there were no beneficial effect of sertraline as a tetracycline helpercompound, despite the previously reported promising in vitro synergy between tetracycline and sertraline.

Nevertheless, the carriage of high level of tetracyclineresistant bacteria among all of the commercial bought piglets used in the study, calls for more prudent antibiotic use in the pig production, new antibiotics or novel efficient helper-compound to re-sensitize bacteria to the traditional antibiotics.

\section{Methods}

\section{Pigs and housing}

Forty-eight healthy female Danish landrace piglets (seven weeks of age) with normal faecal consistency were bought from a commercial pig producer. Unfortunately, data on previous antibiotic treatment for each individual pig was not available. At arrival at the experimental animal unit of University of Copenhagen, Frederiksberg campus the pigs were weighted and randomly allocated to one of four groups, placed in four different pens. Hereafter, the pigs were allowed to acclimatize for one week before starting the trial. The pigs were kept on restricted diet of commercial feed (Svine Erantis Brogaarden ApS, Lynge, Denmark) according to age $(550 \mathrm{~g} / \mathrm{pig} /$ day $)$ and had free access to tap water. The pigs were euthanized by an initially anesthetizing shoot with a captive bolt-pistol followed by incision on jugular vein and bleeding of the pigs. All procedures performed on the pigs were approved and licensed by the Danish Animal Experiments Inspectorate (license no. 2016-15-0201-01144).

\section{Experimental design}

After one week of acclimatization and two days before treatment start (time point $\mathrm{T}[-2]$ ), all pigs were weighted and fecal samples were obtained from all 12 pigs in each of the four groups. Hereafter all pigs were orally inoculated with a tetracycline-resistant indicator strain of E. coli (strain details are given below) in $50 \mathrm{~mL}$ of water-diluted, blended, soft cat food (supermarket brand), which the pig found highly palatable. Inoculation was thus voluntary and stress-free for all pigs, which ensured that all pigs be equally and heavily colonized with a rifampicin-marked tetracycline resistant strain of $E$. coli. Two days after inoculation and just prior to treatment start (time point $\mathrm{T}[0]$ ), all pigs were weighted and individual fecal samples were obtained. Two randomly chosen pigs from each group were hereafter euthanized, and ileum samples were obtained from these eight pigs to represent the ileal microbiota composition before treatment. At the same day, the pigs in Group 2 (tetracycline treated), Group 3 (sertraline treated) and Group 4 (sertraline/ tetracycline treated) received individual, voluntary oral treatment (sertraline or compounds dissolved in blended cat food) for five consecutive days (Doxylin@ (Dopharma Research, Raamsdonksveer, Netherlands) $(24 \mathrm{mg} / \mathrm{kg}$ body weight corresponding to $12.5 \mathrm{mg}$ doxycycline (a tetracycline antibiotic)/kg body weight; Sertrone $\odot$ (KRKA Sverige, Stockholm, Sweden) (one $100 \mathrm{mg}$ sertraline-containing tablet daily, corresponding to approximately four $\mathrm{mg} / \mathrm{kg}$ body weight). Pigs in Group 1 remained un-medicated, but received the same amount $(10 \mathrm{ml})$ of the water-diluted blended cat food. During the treatment period, pigs in Group 2 and 4 were weighted each day to adjust the amount of Doxylin per pig per kg body weight per day. Forty-eight hours after ended treatment period (time point T[7]), fecal samples were obtained from all pigs and hereafter five pigs in each group were chosen randomly and euthanized followed by obtaining of ileum samples from all euthanized pigs. The remaining five pigs in each group remained un-medicated the following nine days post treatment. Nine days after ended treatment (time point $\mathrm{T}[15]$ ), individual fecal samples were obtained, pigs were euthanized and ileum samples were collected from all pigs as well. All pigs were clinically healthy throughout the study period.

Fecal samples were obtained by either spontaneously defecation without the sample reaching the floor or obtained directly from rectum. All samples were collected with sterile latex gloves.

Ileum samples were obtained by localising the ostium ileocecale and hereafter measuring five $\mathrm{cm}$ in the oral direction. At this location a sterile pean was placed to occlude the intestinal lumen. Another $10 \mathrm{~cm}$ in the oral direction were measured and a second occlusive pean placed to allow resection of the ileal segment. The section was placed on a disinfected table and cut open to expose the mucosal surface. A sterile glass microscope slide was used to scrape five times into the depth of the mucosal layer. All material hereof, i.e. the mucosal tissue and intestinal content, was transferred to a sterile petri dish.

All samples were processed (serial dilution plating or DNA extraction) within the same day the samples had been collected.

\section{Preparation of inoculum of indicator bacteria}

The E. coli tetracycline resistant " $E$. coli O2" was chosen as an indicator strain because in vitro synergy with sertraline has been documented and investigated in detail [10]. Secondly, the strain does not contain any of the primary virulence factors associated with porcine enterotoxigenic E. coli (toxins, fimbria F4/F18 ect.) [27]. The strain contains two large plasmids; one encoding resistances toward seven different antimicrobials, including tetracycline [28], and a virulence plasmid [29], genes encoding for increased survival in extra-intestinal compartments, e.g. the human urinary tract system [30]. For re-isolation purposes, rifampicin resistance was induced by standard procedures. The rifampicin mutant strain 
was confirmed to have the same tetracycline resistance properties and synergy with tetracycline as previously reported for the wild-type strain of E. coli $\mathrm{O} 2$ [10].

The strain had been stored at $-80^{\circ} \mathrm{C}$ in Brain and Heart Infusion (BHI) broth (Oxoid, Basingstoke, UK) in $15 \%(v / v)$ glycerol until needed. The day before inoculation four colonies of the strain were picked from an agar plate (Oxoid, CM0055) supplemented with 5\% calf blood. Each colony was inoculated into a flask containing $250 \mathrm{~mL}$ BHI broth and incubated at $37^{\circ} \mathrm{C}$ without shaking for $24 \mathrm{~h}$ to reach a concentration of approximately $10^{9} \mathrm{CFU}$. The four overnight grown cultures were pooled to one culture in a sterile Blue cap bottle (Sigma-Aldrich, Copenhagen, Denmark) and distributed into $50 \mathrm{~mL}$ centrifuge tubes each containing $40 \mathrm{~mL}$ of the pooled culture and centrifuged at $4{ }^{\circ} \mathrm{C}$ at $3000 \mathrm{G}$ for $15 \mathrm{~min}$. Subsequently, the supernatant in each tube was discharged and the pellet re-suspended in $10 \mathrm{ml}$ phosphate buffered solution (PBS), and again pooled together in one pool, of which each pig orally received five $\mathrm{ml}$. To determine the exact dose administered to each pig, $100 \mu \mathrm{l}$ of the pooled, re-suspended bacterial solution was used to make a 10-fold serially dilutions until $10^{-11}$ dilution and from each dilution, $100 \mu \mathrm{l}$ was plated on $\mathrm{MH}$ ager (Oxoid Ltd., Thermo Scientific, Roskilde, Denmark) to determine the final inoculation dose of $2 \times 10^{12} \mathrm{CFU}$ per pig.

\section{Bacterial quantification}

Approximately one gram of each sample (fecal or ileum was diluted 1:10 in PBS in a Biomaster $80 \odot$ filter bag (Stomacher, Seward Inc., Port Saint Lucie, FL, USA) and homogenized in a Stomacher machine (Stomacher, Seward Inc) for $1 \mathrm{~min}$. Subsequently, serial 10-fold dilutions were made in $0.9 \% \mathrm{NaCl}$ solution. From all dilutions $\left(10^{-1}\right.$ to $\left.10^{-7}\right), 10 \mu \mathrm{l}$ were spotted on agar plates containing the following two media: MacConkey agar supplemented with $8 \mathrm{mg} / \mathrm{L}$ tetracycline (for enumeration of total tetracycline resistant coliforms), and MacConkey agar supplemented with $8 \mathrm{mg} / \mathrm{L}$ tetracycline, $50 \mathrm{mg} / \mathrm{L}$ ampicillin (Sigma-Aldrich) and $25 \mathrm{mg} /$ liter rifampicin (Sigma-Aldrich) (For enumeration of the indicator strain E. coli).

E. coli K-12 MG1655 (tetracycline sensitive) and E. coli NCTC 50078 (tetracycline resistant) were included as control strains on all plates. For MacConkey agar supplemented with tetracycline, rifampicin and ampicillin, the inoculum strain (E. coli_O2 [31]) was included as well.

All serial dilutions were done and plated in duplicates. The plates were aerobically incubated for $24 \mathrm{~h}$ at $37^{\circ} \mathrm{C}$ followed by colony enumeration.

For each plate, a count, expressed as the number of CFU per gram, was determined using a weighed arithmetic mean based on the two highest dilutions showing the separation between colonies, and finally, the number of CFU per gram was $\log 10$ transformed. To avoid exclusion of samples with a CFU per gram equaling zero from the analysis (as 0 cannot be $\log _{10}$ transformed), all numbers of CFU per gram sample, was added a constant of 1. This constant was chosen because $\log 10$ to 1 equals 0 .

\section{PCR confirmation of re-isolation of inoculated indicator strain E. coli_O2}

To verify that the isolates from fecal or ileal samples growing on the agar plate containing MacConkey supplemented with tetracycline, rifampicin and ampicillin plate were identical to the inoculum strain of E. coli, two randomly chosen isolates from each pig were confirmed as identical to the inoculum strain by an E. coli_O2 specific PCR as described in Kromann et al. (2017) [32].

\section{DNA extraction of fecal and ileum samples}

Total DNA from 48 ileal and10 fecal samples, respectively, were extracted for determination of the microbiota composition. From the $10^{-1}$ diluted homogenates used for the enumeration of bacteria, $800 \mu \mathrm{l}$ homogenate from each of the samples was transferred to a $2 \mathrm{~mL}$ FastPrep $\odot$ Lysis matrix E tube (MP Biomedical, Solon, OH, USA) and further homogenized on a FastPrep-24 Instrument (MP Biomedical) at $6 \mathrm{~m} / \mathrm{sec}$ for $40 \mathrm{~s}$, followed by centrifugation for $15 \mathrm{~min}$ at $10000 \mathrm{G}$. Subsequently, $400 \mu \mathrm{l}$ of the supernatant of each sample were processed on a Maxwell@ RSC instrument (Promega Corporation, Mannheim, Germany) applying the RSC PureFood GMO kit (Cat. \# AS1600) according to manufacturer's protocol for extraction of DNA.

DNA concentrations were measured on an Agilent 2100 Bioanalyzer (Agilent Technologies, Waldbronn, Germany) before $16 \mathrm{~S}$ rRNA gene amplicon library preparation.

\section{S rRNA gene amplicon library preparation}

Bacterial V3-4 16S rRNA gene sequencing libraries of extracted DNA from 48 ileal samples and 10 fecal samples were prepared by a custom protocol based on an Illumina (Illumina, San Diego, CA, USA) protocol [33] according to previously done by Olsen et al. 2017 [34]. Briefly, for PCR amplification of the 16S rRNA gene fragments, $10 \mathrm{ng}$ of extracted DNA was used as template. Each PCR reaction $(25 \mu \mathrm{L})$ contained dNTPs $\left(100 \mu \mathrm{M}\right.$ of each), MgSO4 (1.5 mM), Platinum ${ }^{\circ} \mathrm{Taq}$ DNA polymerase HF (2 mU), 1X Platinum ${ }^{\circ}$ High Fidelity buffer (Thermo Fisher Scientific, USA) and tailed primer mix ( $400 \mathrm{nM}$ of each forward and reverse). PCR conditions included: An nitial denaturation at $95^{\circ} \mathrm{C}$ for $2 \mathrm{~min}$, 35 cycles of amplification $\left(95^{\circ} \mathrm{C}\right.$ for $20 \mathrm{~s}, 50^{\circ} \mathrm{C}$ for $30 \mathrm{~s}$, $72^{\circ} \mathrm{C}$ for $60 \mathrm{~s}$ ) and a final elongation at $72^{\circ} \mathrm{C}$ for $5 \mathrm{~min}$. Duplicate PCR reactions was performed for each sample and the duplicates were pooled after PCR. The forward and reverse tailed primers were designed according to 
Vo and Jedlicka [33] and contain a primer parts targeting the respective 16S rRNA gene fragments. Bacteria V3-4 [35]: 5'-CCTACGGGNGGCWGCAG (341F) and 5'-GACTACHVGGGTATCTAATCC (805R). The primer tails enable attachment of Illumina Nextera adaptors for sequencing in a subsequent PCR. The amplicon libraries were purified using Agencourt Ampure XP Bead (Beckman Coulter) using vendor recommended protocol, using a bead to sample ratio of 4:5 and the DNA was eluted in $33 \mu \mathrm{L}$ of nuclease free water (Qiagen, Hilden, Germany). DNA concentration was measured using Quant-iT DNA Assay Kit, high sensitivity (Thermo Fisher Scientific, Roskilde, Denmark). Sequencing libraries were prepared from the purified amplicon libraries using a second PCR. Each PCR reaction $(25 \mu \mathrm{L})$ contained 1x PCRBIO HiFi buffer (PCR Biosystems Ldt, London, UK), PCRBIO HiFi Polymerase (1 U) (PCR Biosystems Ldt), adaptor mix ( $400 \mathrm{nM}$ of each forward and reverse) and $2 \mu \mathrm{L}$ of amplicon library template. PCR was run with the following program: Initial denaturation at $95^{\circ} \mathrm{C}$ for $2 \mathrm{~min}, 8$ cycles of amplification $\left(95^{\circ} \mathrm{C}\right.$ for $20 \mathrm{~s}$, $55^{\circ} \mathrm{C}$ for $30 \mathrm{~s}, 72^{\circ} \mathrm{C}$ for $60 \mathrm{~s}$ ) and a final elongation at $72^{\circ} \mathrm{C}$ for $5 \mathrm{~min}$. The sequencing libraries were purified using Agencourt Ampure XP Bead (Beckman Coulter, Redlands, CA, USA) using vendor recommended protocol, using a sample/bead ratio of 5:4 and the DNA was eluted in $20 \mu \mathrm{L}$ of nuclease free water (Qiagen). DNA concentration was measured using Quant-iT DNA Assay Kit, high sensitivity (Thermo Fisher Scientific). Gel electrophoresis using Tapestation 2200 and D1000 High Sensitivity screentapes (Agilent Technologies) was used to check the product size and purity of randomly picked sequencing libraries.

\section{DNA sequencing}

The purified sequencing libraries were pooled in equimolar concentrations and diluted to $4 \mathrm{nM}$. The samples were paired end sequenced ( $2 \times 301 \mathrm{bp}$ ) on a MiSeq (Illumina) using a MiSeq Reagent kit v3, 600 cycles (Illumina) following the standard guidelines for preparing and loading samples on the MiSeq. 20\% Phix control library was spiked in to overcome low complexity issue often observed with amplicon samples.

\section{$16 \mathrm{~S}$ rRNA gene amplicon bioinformatic processing}

Forward and reverse reads were trimmed for quality using the software Trimmomatic v. 0.32 [36] with the settings SLIDINGWINDOW:5:3 and MINLEN:275. The trimmed forward and reverse reads were merged using the program FLASH v. 1.2.7 [37], with the settings -m $25-\mathrm{M} 200$. The merged reads were dereplicated and formatted for use in the UPARSE workflow [38]. The dereplicated reads were clustered, using the usearch $\mathrm{v}$. 7.0.1090 -cluster_otus command with default settings.
Operational Taxanomic Units (OUT) abundances were estimated using the usearch v. 7.0.1090 -usearch_global command with -id 0.97. Taxonomy was assigned using the RDP classifier [39] as implemented in the paralle1_assign_taxonomy_rdp.py script in QIIME [40], using the MiDAS database v.1.23 [41]. The results were analysed in R [42] through the Rstudio IDE using the ampvis package v.2.0 [43].

\section{Average weight gain}

The average weight gain per pig was calculated as difference in body weight from the day of arrival to the experimental unit compared to the body weight of the pig at the day of euthanization divided by the days spend at the experimental unit.

\section{Statistical analysis}

Statistical analyses were done using the software Graphpad Prism version 7 (Graphpad Software, Inc., La Jolla, CA, USA). At each time sampling point, differences between groups for $\log 10 \mathrm{CFU}$ on the five different types of agar plates, proportions of CFU counts, and abundances of different genera in the microbiota or average weight gain/group were analysed by one-way ANOVA. In addition, differences within each group at different sampling times were analysed in the same manner. The one-way ANOVA was followed by Turkey's t-test for multiple comparisons. A statistical difference of $P<0.5$ was considered statistically significant.

\section{Additional files}

\begin{abstract}
Additional file 1: Table S1. Sample concentration and number of reads after sequencing. "Reads" is the number of reads after sequencing after sequencing, quality control and bioinformatics processing, "Observed" is the number of observed operational taxonomic units in 10000 reads, while Shannon is the Shannon index observed in 10000 reads. (DOCX 19 $\mathrm{kb})$
\end{abstract}

Additional file 2: Figure S2. The 25 most abundant families genera. The overall 25 most abundant genera in percent. Each has both a broad name (Phylum) and a specific name (Genus). Samples from the untreated control group ( $)$, sertraline treated (S), tetracycline (T) and sertraline/tetracycline treated groups were obtained (ST) (D0/T[0]), $48 \mathrm{~h}$ after last day of treatment (D1/T[7]) and nine days after last day of treatment (D9/T[15]). Feces samples were only obtained nine days after ended treatment just prior to treatment start. From the latter two, samples P01, P06, P07, P08 and P09 were obtained from pigs in the un-medicated control group, while samples P14, P17, P19, P20 and P22 were obtained from pigs that had received tetracycline treatment. (JPG $149 \mathrm{~kb}$ )

Additional file 3: Figure S1. Principle component analysis (PCA) of ileum microbiota composition at time T[0] and T[15]. Identification of samples with similar microbial communities using multivariate statistics (PCA). Each red or blue point represent the microbita composition in a specific sample obtained just prior to treatment start (D0/T[0]) or nine days after the last day of treatment (D9/T[15]), respectively. (JPG $42 \mathrm{~kb}$ )

\section{Abbreviations}

CFU: Colony forming units; OUT: Operational Taxanomic Units;

PCR: Polymerase Chain Reaction; SSRI: Selective Serotonin Reuptake Inhibitor 


\section{Acknowledgments}

Technician Dan Ryttov is thanked for skillful laboratory assistance. DNAsense is thanked for the high-quality sequencing of 16S rDNA.

\section{Funding}

This project has received funding from the Danish Council of Independent Research (DCIR) grant agreement no. 4184-00512. The DCRI did not participate in designing the study, the analysis of and interpretation of data, nor in writing the manuscript.

\section{Availability of data and materials}

All data are available by request to the corresponding author.

\section{Authors' contributions}

All authors contributed to the design of the experiment. SK, RHO, DBS and $\mathrm{AH}$ conducted the experimental treatment of pigs, sampling, bacterial cultivation and DNA extractions. $\mathrm{RHO}$ prepared samples for metagenomic sequencing. $\mathrm{RHO}, \mathrm{MB}, \mathrm{SJ}$ and JPN prepared the manuscript. All authors contributed to critical revision of the manuscript. All authors read and approved the final manuscript.

\section{Ethics approval and consent to participate}

All procedures performed on the pigs were approved and licensed by the Danish Animal Experiments Inspectorate (license no. 2016-15-0201-01144).

\section{Consent for publication}

Not applicable.

\section{Competing interests}

The authors declare that they have no competing interests.

\section{Publisher's Note}

Springer Nature remains neutral with regard to jurisdictional claims in published maps and institutional affiliations.

\section{Received: 15 October 2017 Accepted: 27 December 2018}

\section{Published online: 11 January 2019}

\section{References}

1. The Danish Integrated Antimicrobial Resistance Monitoring and Research Programme (DANMAP). 2012

2. Sengelov G, Halling-Sorensen B, Aarestrup FM. Susceptibility of Escherichia coli and enterococcus faecium isolated from pigs and broiler chickens to tetracycline degradation products and distribution of tetracycline resistance determinants in E. coli from food animals. Vet Microbiol. 2003:95:91-101.

3. Zhang Y, Boyd SA, Teppen BJ, Tiedje JM, Li H. Role of tetracycline speciation in the bioavailability to Escherichia coli for uptake and expression of antibiotic resistance. Environ Sci Technol. 2014;48:4893-900.

4. Thaker M, Spanogiannopoulos P, Wright GD. The tetracycline resistome. Cell Mol Life Sci. 2010;67:419-31.

5. Burow E, Simoneit C, Tenhagen BA, Kasbohrer A. Oral antimicrobials increase antimicrobial resistance in porcine E. coli--a systematic review. Prev Vet Med. 2014;113:364-75.

6. Van Boeckel TP, Brower C, Gilbert M, Grenfell BT, Levin SA, Robinson TP, Teillant A, Laxminarayan R. Global trends in antimicrobial use in food animals. Proc Natl Acad Sci U S A. 2015;112:5649-54.

7. Martins M, Dastidar SG, Fanning S, Kristiansen JE, Molnar J, Pages JM, Schelz Z, Spengler G, Viveiros M, Amaral L. Potential role of non-antibiotics (helper compounds) in the treatment of multidrug-resistant gram-negative infections: mechanisms for their direct and indirect activities. Int J Antimicrob Agents. 2008;31:198-208.

8. Nikaido $\mathrm{H}$. Prevention of drug access to bacterial targets: permeability barriers and active efflux. Science. 1994:264:382-8.

9. Ayaz M, Subhan F, Ahmed J, Khan AU, Ullah F, Ullah I, Ali G, Syed NI, Hussain S. Sertraline enhances the activity of antimicrobial agents against pathogens of clinical relevance. J Biol Res (Thessalon ). 2015;22:4.

10. Li L, Kromann S, Olsen JE, Svenningsen SW, Olsen RH. Insight into synergetic mechanisms of tetracycline and the selective serotonin reuptake inhibitor, sertraline, in a tetracycline-resistant strain of Escherichia coli. J Antibiot (Tokyo). 2017;70:944-53.
11. Munoz-Bellido JL, Munoz-Criado S, Garcia-Rodriguez JA. In-vitro activity of psychiatric drugs against Corynebacterium urealyticum (Corynebacterium group D2). J Antimicrob Chemother. 1996;37:1005-9.

12. Amlesh S, Chattopadhyayb D, Sinha C, Janaa A, Ghosha S, Banerjeea A, Hendricks O, Christensen J, Kristiansen J. Evaluation of In Vivo and In Vitro antimicrobial activities of a selective serotonin reuptake inhibitor sertraline hydrochloride. Anti-Infective Agents. 2012;10:95-104.

13. Bohnert JA, Szymaniak-Vits M, Schuster S, Kern WV. Efflux inhibition by selective serotonin reuptake inhibitors in Escherichia coli. J Antimicrob Chemother. 2011;66:2057-60.

14. Anonym. Regulation $1831 / 2003 /$ EC on additives for use in animal nutrition. In: Replacing directive 70/524/EEC on additives in feeding-stuffs; 2003.

15. Madec F, Bridoux N, Bounaix S, Cariolet R, Duval-Iflah Y, Hampson DJ, Jestin A. Experimental models of porcine post-weaning colibacillosis and their relationship to post-weaning diarrhoea and digestive disorders as encountered in the field. Vet Microbiol. 2000;72:295-310.

16. Weber N, Nielsen JP, Jakobsen AS, Pedersen LL, Hansen CF, Pedersen KS. Occurrence of diarrhoea and intestinal pathogens in non-medicated nursery pigs. Acta Vet Scand. 2015;57:64.

17. Boumba VA, Rallis G, Petrikis P, Vougiouklakis T, Mavreas V. Determination of clozapine, and five antidepressants in human plasma, serum and whole blood by gas chromatography-mass spectrometry: a simple tool for clinical and postmortem toxicological analysis. J Chromatogr B Analyt Technol Biomed Life Sci. 2016;1038:43-8.

18. Gobin V, Van SK, Denys D, Deforce D. Selective serotonin reuptake inhibitors as a novel class of immunosuppressants. Int Immunopharmacol. 2014;20: 148-56.

19. Kanwar N, Scott HM, Norby B, Loneragan GH, Vinasco J, Cottell JL, Chalmers G, Chengappa MM, Bai J, Boerlin P. Impact of treatment strategies on cephalosporin and tetracycline resistance gene quantities in the bovine fecal metagenome. Sci Rep. 2014;4:5100.

20. Simoneit C, Burow E, Tenhagen BA, Kasbohrer A. Oral administration of antimicrobials increase antimicrobial resistance in E. coli from chicken-a systematic review. Prev Vet Med. 2015;118:1-7.

21. Vieira AR, Houe $H$, Wegener HC, Lo Fo Wong DM, Emborg HD. Association between tetracycline consumption and tetracycline resistance in Escherichia coli from healthy Danish slaughter pigs. Foodborne Pathog Dis. 2009:6:99-109.

22. Stenger M, Behr-Rasmussen C, Klein K, Gronnemose RB, Andersen TE, Klitgaard JK, Kolmos HJ, Lindholt JS. Systemic thioridazine in combination with dicloxacillin against early aortic graft infections caused by Staphylococcus aureus in a porcine model: in vivo results do not reproduce the in vitro synergistic activity. PLoS One. 2017;12:e0173362.

23. Chopra I, Roberts M. Tetracycline antibiotics: mode of action, applications, molecular biology, and epidemiology of bacterial resistance. Microbiol Mol Biol Rev. 2001;65:232-60

24. Graesboll K, Damborg P, Mellerup A, Herrero-Fresno A, Larsen I, Holm A Nielsen JP, Christiansen LE, Angen O, Ahmed S, Folkesson A, Olsen JE. Effect of tetracycline dose and treatment mode on selection of resistant coliform Bacteria in nursery pigs. Appl Environ Microbiol. 2017;83.

25. Looft T, Johnson TA, Allen HK, Bayles DO, Alt DP, Stedtfeld RD, Sul WJ, Stedtfeld TM, Chai B, Cole JR, Hashsham SA, Tiedje JM, Stanton TB. In-feed antibiotic effects on the swine intestinal microbiome. Proc Natl Acad Sci U S A. 2012;109:1691-6.

26. Suda Y, Villena J, Takahashi Y, Hosoya S, Tomosada Y, Tsukida K, Shimazu T, Aso H, Tohno M, Ishida M, Makino S, Ikegami S, Kitazawa H. Immunobiotic lactobacillus jensenii as immune-health promoting factor to improve growth performance and productivity in post-weaning pigs. BMC Immunol. 2014;15:24.

27. Melkebeek V, Goddeeris BM, Cox E. ETEC vaccination in pigs. Vet Immunol Immunopathol. 2013;152:37-42

28. Johnson TJ, Siek KE, Johnson SJ, Nolan LK. DNA sequence and comparative genomics of pAPEC-O2-R, an avian pathogenic Escherichia coli transmissible R plasmid. Antimicrob Agents Chemother. 2005;49:4681-8.

29. Johnson TJ, Siek KE, Johnson SJ, Nolan LK. DNA sequence of a ColV plasmid and prevalence of selected plasmid-encoded virulence genes among avian Escherichia coli strains. J Bacteriol. 2006;188:745-58.

30. Skyberg JA, Johnson TJ, Johnson JR, Clabots C, Logue CM, Nolan LK. Acquisition of avian pathogenic Escherichia coli plasmids by a commensal $\mathrm{E}$. coli isolate enhances its abilities to kill chicken embryos, grow in human urine, and colonize the murine kidney. Infect Immun. 2006;74:6287-92. 
31. Jorgensen SL, Kudirkiene E, Li L, Christensen JP, Olsen JE, Nolan L, Olsen RH. Chromosomal features of Escherichia coli serotype O2:K2, an avian pathogenic E. coli. Stand Genomic Sci. 2017;12:33.

32. Kromann S, Kudirkiene E, Li L, Thofner I, Christensen JP, Olsen RH. Treatment with high-dose antidepressants severely exacerbate the pathological outcome of experimental Escherichia coli infections in poultry. PLoS One. In press

33. Vo AT, Jedlicka JA. Protocols for metagenomic DNA extraction and Illumina amplicon library preparation for faecal and swab samples. Mol Ecol Resour. 2014;14:1183-97.

34. Olsen R, Kudirkiene E, Thofner I, Pors S, Karlskov-Mortensen P, Li L, Papasolomontos S, Angastiniotou C, Christensen J. Impact of egg disinfection of hatching eggs on the eggshell microbiome and bacterial load. Poult Sci. 2017;96:3901-11.

35. Herlemann DP, Labrenz M, Jurgens K, Bertilsson S, Waniek JJ, Andersson AF. Transitions in bacterial communities along the $2000 \mathrm{~km}$ salinity gradient of the Baltic Sea. ISME J. 2011;5:1571-9.

36. Bolger AM, Lohse M, Usadel B. Trimmomatic: a flexible trimmer for Illumina sequence data. Bioinform. 2014;30:2114-20.

37. Magoc T, Salzberg SL. FLASH: fast length adjustment of short reads to improve genome assemblies. Bioinform. 2011;27:2957-63.

38. Edgar RC. UPARSE: highly accurate OTU sequences from microbial amplicon reads. Nat Methods. 2013;10:996-8.

39. Wang Q, Garrity GM, Tiedje JM, Cole JR. Naive Bayesian classifier for rapid assignment of rRNA sequences into the new bacterial taxonomy. Appl Environ Microbiol. 2007:73:5261-7.

40. Caporaso JG, Kuczynski J, Stombaugh J, Bittinger K, Bushman FD, Costello EK, Fierer N, Pena AG, Goodrich JK, Gordon Jl, Huttley GA, Kelley ST, Knights D, Koenig JE, Ley RE, Lozupone CA, McDonald D, Muegge BD, Pirrung M, Reeder J, Sevinsky JR, Turnbaugh PJ, Walters WA, Widmann J, Yatsunenko T, Zaneveld J, Knight R. QIIME allows analysis of high-throughput community sequencing data. Nat Methods. 2010;7:335-6.

41. Mcllroy SJ, Saunders AM, Albertsen M, Nierychlo M, Mcllroy B, Hansen AA, Karst SM, Nielsen JL, Nielsen PH. MiDAS: the field guide to the microbes of activated sludge. Database (Oxford). 2015;2015:bav062.

42. Core Team R. A language and environment for statistical computing. R Foundation for statistical computing. Austria: Core Team R; 2015.

43. Albertsen M, Karst SM, Ziegler AS, Kirkegaard RH, Nielsen PH. Back to basics - the influence of DNA extraction and primer choice on phylogenetic analysis of activated sludge communities. PLoS One. 2015;10:e0132783.

Ready to submit your research? Choose BMC and benefit from:

- fast, convenient online submission

- thorough peer review by experienced researchers in your field

- rapid publication on acceptance

- support for research data, including large and complex data types

- gold Open Access which fosters wider collaboration and increased citations

- maximum visibility for your research: over $100 \mathrm{M}$ website views per year

At $\mathrm{BMC}$, research is always in progress.

Learn more biomedcentral.com/submissions 